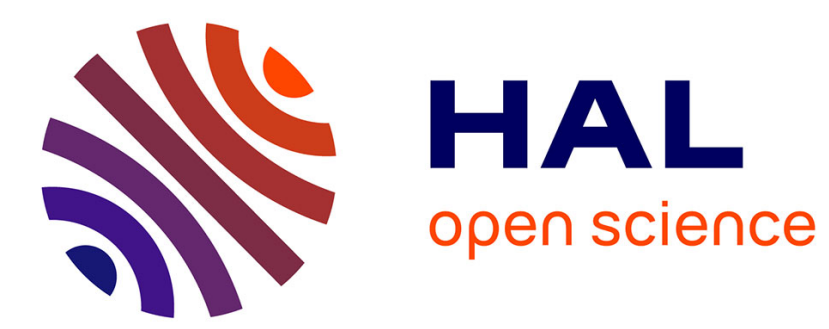

\title{
Le sacré et la recherche du Dieu révélé selon Romano Guardini
}

Grégory Woimbée

\section{To cite this version:}

Grégory Woimbée. Le sacré et la recherche du Dieu révélé selon Romano Guardini. Transversalités, 2016. hal-02494783

\section{HAL Id: hal-02494783 \\ https://hal.science/hal-02494783}

Submitted on 3 Mar 2020

HAL is a multi-disciplinary open access archive for the deposit and dissemination of scientific research documents, whether they are published or not. The documents may come from teaching and research institutions in France or abroad, or from public or private research centers.
L'archive ouverte pluridisciplinaire HAL, est destinée au dépôt et à la diffusion de documents scientifiques de niveau recherche, publiés ou non, émanant des établissements d'enseignement et de recherche français ou étrangers, des laboratoires publics ou privés. 


\section{Le sacré et la recherche du Dieu révélé selon Romano Guardini}

\section{Grégory Woimbée}

Cette communication ${ }^{1}$ se propose bien modestement d'envisager la question du sacré à partir de la réflexion de Romano Guardini (1885-1968) sur l'expérience religieuse. Pour lui, l'ancienne culture, la "moderne», touche à sa fin, et le christianisme s'en trouve comme désenveloppé, mis à nu. Dans ce dialogue contemporain de la foi avec la culture, Guardini réhabilite le sacré. Ambigu et ambivalent, le sacré est pourtant un socle du divin préparant à la relation personnelle au divin.

\section{I - Le sacré, comme crise de l'expérience religieuse}

Guardini part d'une possibilité que la raison moderne n'a pas sérieusement envisagée, sans l'avoir nécessairement écartée : le fait que Dieu existe. Et si l'attitude religieuse répondait à une réalité donnée, et s'il y avait autre chose entre la chose et l'œil qui la voit ? Dès lors qu'il regarde, l'homme est saisi dans une relation primordiale qui n'est ni l'œil qui voit ni l'objet qui est vu' ${ }^{2}$. La foi est, chez Gardini, l'événement d'une rencontre. Sur le plan de ce que vit l'homme croyant, le sacré et la foi ne sont pas séparés. Sa Weltanschaunng, sa vision du monde, n'est pas seulement une relation dans la raison mais une relation dans l'existence. Ce que les sociologues ont pensé comme une séparation, entre sacré-non profane et profane-non sacré, Guardini le pense comme une jonction. Le phénomène religieux est par nature incirconscrit du point de vue de la norme religieuse. Le sacré n'est pas tout le religieux, mais ce qui porte, dans ce qui n'est pas divin, le dynamisme de la rencontre avec le divin.

Guardini distingue dans l'expérience religieuse une expérience religieuse naturelle (le sacré) et une expérience religieuse personnelle (la foi). Il ne sépare pas l'essence du rapport de l'homme à Dieu des conditions de sa variabilité historique, mais veut souligner un point de crise. La sécularisation ou la désacralisation est une crise de l'expérience religieuse naturelle. Lorsqu'il s'intéresse au sacré, Guardini le fait pour en constater la crise, car il ne le conçoit pas comme expérience religieuse close sur elle-même ou complète, mais plutôt comme un concept hypothétique qui ne se révèle véritablement que dans le mouvement de la foi. Par le sacré, l'homme découvre qu'il ne s'atteint pas lui-même par lui-même. La crise religieuse consiste pour l'homme à exprimer la nostalgie de son origine religieuse.

L'homme est un animal symbolique, il ne croit pas sans signe, ni sans geste, ni sans mot. Cela tient au fait de la création et à ce que Rudolph Otto a appelé le champ du numineux, cette sphère qui désigne ce qui se tient au-delà de l'éthique et du rationnel, et qui se présente sous la forme ambivalente du mystère effrayant et fascinant. L'immatériel comporte des expériences à la fois non rationnelles et non absurdes. La religiosité immanente ou "naturelle ", avant même toute construction mentale, sociale ou culturelle, tient en ce qu'une « vague venant de Dieu vient faire ressac contre ce tracé qui est au plus profond de moi-même, tracé au-delà duquel il y a tout ce qui est autre ${ }^{3}$.» Elle n'est pas seulement relation à l'absolue, mais relation animée à l'absolu : l'homme concret vit déjà du Dieu vivant. La religion naturelle est déjà une relation implicitement personnelle (l'homme est toujours devant Dieu), sans être une relation personnelle accomplie, c'est-à-dire interpersonnelle.

\footnotetext{
${ }^{1}$ Romano Guardini, Journée d'étude du 5 novembre 2013, Institut Catholique de Paris (avec, par ordre d'intervention, Jean Greisch, Ugo Perone, Grégory Woimbée et Jérôme de Gramont).

${ }^{2}$ Cf. Woimbée, G., L'esprit du christianisme. Introduction à la pensée de Romano Guardini, Genève, Ad Solem, voir chap.4 : Libérer la raison par la folie de l'œil, p. 93-106.

${ }^{3}$ Guardini, R., Lettres du Lac de Côme, Paris, Cerf, 1955 (or. 1926), p.201.
} 
Quelle est la fonction du sacré dans le dynamisme religieux de la foi ? Pour Guardini, le sacré constitue une forme de lisibilité de la Révélation divine qui est l'événement historique de l'auto-ouverture de Dieu qui a culminé dans l'incarnation de la Parole de Dieu, dans le Verbe fait chair. Dieu révèle Dieu, non sous la forme de doctrines, mais sous la forme d'une action, action qui supporte l'enseignement de Dieu lui-même, c'est-à-dire de son auto-communication. ${ }^{4}$ Les hommes ne viennent pas à Dieu d'abord par l'enseignement de l'Eglise, aussi nécessaire soit-il, mais par la rencontre avec le Christ vivant en son Eglise, Guardini ajoutant « dans l'Esprit et par l'Eglise ». La Révélation divine, si elle est une action de Dieu du point de vue de l'homme, prend la forme d'une action de l'homme du point de vue de Dieu. Guardini la nomme conversion. La Révélation n'est donc pas un système abstrait de doctrines ou de valeurs, elle est un agir divin et humain. Il faut donc qu'elle soit vécue pour être connue. Le sacré lui fournit un langage et constitue l'univers dans lequel l'homme interprète la Révélation de Dieu dans l'histoire.

\section{II - La foi face à la perte du sacré}

Face à la perte du sacrée, car la crise du sacré est une perte, puisque le sacré est un élément fondateur, que peut-on faire? Guardini commence par découvrir ce qui a provoqué cette perte. C'est pour lui l'affaiblissement de ce qu'il appelle «l'organe religieux », par lequel le religieux est perçu, identifié, reconnu et rencontré comme tel. Cette intimité blessée de l'bomo religiosus ne lui fait plus sentir le religieux : devant lui, les choses ne dégagent plus rien de religieux, plus rien qui le dessaisit de lui-même, plus rien à contempler, car contempler c'est se laisser regarder par l'objet visé, se laisser transformer par le regard de l'Autre qui passe à travers l'objet visé. L'immédiateté de la puissance, l'idéologie de la main mise, le règne de la technique rompent les médiations ordinaires par lesquelles l'homme perçoit le sens de ce qui est, sur le mode d'un ravissement et non d'une conquête : l'intersubjectivité, l'art, le symbole, le langage et la personne sont devenues des valeurs marchandes. L'agonie du sacré touche la foi parce qu'elle atteint l'homme, ce qui se passe en lui et ce qu'il fait de ce qui se tient devant lui. Et la foi sans culture, sans la condensation des représentations et des significations historiques que l'homme se fait de son rapport au réel, et la foi sans la raison, sans la compréhension de sa propre expérience du réelle, cette foi verse dans le fondamentalisme, incline à supprimer les médiations qui le ravissent à lui-même et à sa violence.

Guardini n'entend pas proposer un plan de reconstitution du sacré. Il envisage le devenir de la foi et la forme historique qu'elle prendra. Si la foi ne change pas substantiellement, sa conscience évolue nécessairement. Pour Guardini, c'est une conscience beaucoup plus mystique qui se développera, et donc beaucoup plus élitiste et minoritaire. Dans l'expérience religieuse, on distingue l'ascèse (commune au plus grand nombre et consistant dans les choses à faire pour se rendre disponible à Dieu) et la mystique (privilège d'un petit nombre et consistant à vivre totalement abandonné et uni à Dieu). Ce courant élitiste est le fait de nombreux théologiens qui ont toujours perçu d'un œil suspicieux le christianisme de masse et sa reprise de l'idée impériale sous la forme historique d'une chrétienté romano-franque et romano-byzantine. Certains, ce qui n'est pas le cas de Guardini, voient même dans l'exclusion sociale et culturelle du christianisme en Occident, l'occasion de voir émerger une forme plus haute de christianisme, pure, utopique, ukronique dirait Paul Ricœur, rêvée par les Lumières et qui fait penser à ce que Péguy disait du kantisme : «Il a les mains pures, mais il n'a pas de mains. »

La poésie est le révélateur le plus intime et profond de ce qui se passe. Derrière l'apparente stabilité du dogme et de l'institution, la littérature montre la perte progressive du sens du sacré dans la culture occidentale. Guardini l'a bien compris. Il retrace une histoire de la pensée occidentale moderne à travers la figure sécularisée de l'ange. Loin de la Divine Comédie et de la vision de Dante, Hölderlin commence la sécularisation que Rilke achève et conduit à son sommet esthétique. Le messager de Dieu, le médiateur de la parole divine devient « l'oiseau de l'âme » qui appartient à

\footnotetext{
${ }^{4}$ Guardini, R., Die Offenbachung : Ibr Wesen une Ibre Formen, Würzburg, Werknind-Verlag, 1940.
} 
l'espace intérieur de l'âme et aux étoiles, ne différant en rien de l'homme, mais se tenant loin de lui, inaccessible humain, mais inexistant divin.

Pour Guardini la foi donne au croyant de rester ferme dans le péril de son existence, lorsque les rouages de sa vie s'effondrent. Sombre, sans doute imprégné par les effets du fléau totalitaire : "Sans l'élément religieux, la vie devient comme un moteur qui n'a plus d'huile. Il chauffe. A tout instant telle ou telle pièce brûle. Partout des rouages se démontent qui devraient exactement s'emboîter. Le centre et les attaches se rompent. L'existence se désorganise et alors se produit ce court-circuit qui s'accomplit depuis trente ans [il écrit en 1950] dans une mesure toujours croissante. On use de la violence et c'est par là que la détresse, impuissante, cherche une issue. Quand les hommes ne se sentent plus unis de l'intérieur, on les organise extérieurement, et pour que l'organisation travaille, l'Etat met par derrière sa contrainte. Mais peut-on, à la longue, vivre par contrainte ${ }^{5}$ ?»

Il ajoute même: "La solitude de la foi sera terrible. L'amour, la charité disparaîtra du comportement général du monde ${ }^{6} . »$

\section{III - La foi mise à nue}

Cette foi a une vertu essentielle: "Elle a le sens de l'humour. L'humour dans la vie religieuse est une chose merveilleuse ; c'est un humour qui englobe tout ce qui est humain, même l'insuffisance et le singulier, dans l'infini de la bonté de Dieu, qui espère une solution là où la raison et l'énergie n'en voient plus, qui pressent un sens où le sérieux et le zèle y ont depuis longtemps renoncé. $»^{7}$ Cet humour du croyant n'est pas le cynisme blasé qui n'attend plus rien ni personne, c'est l'ultime confiance en Dieu. Pour Kierkegaard, l'humour est ce qui permet de sauvegarder l'incognito du religieux, son « nécessaire » échec : le succès religieux qui est la sainteté se rit de toute justification conceptuelle ou existentielle. Lorsque Guardini parle de l'humour comme moyen de soutenir le doute, il le fait à Berlin dans une homélie prononcée en pleine époque nazie. L'humour s'apparente à la fois au courage qui lui donne sa forme et à la lucidité qui constitue son essence.

Si la crise du sacré éclaire l'acte non-croyant. Elle montre que la non-croyance n'est pas seulement l'antithèse de la foi ou son absence en tant qu'elle naitrait d'un refus ou d'une impossibilité de croire, elle n'est pas seulement athéisme ou agnosticisme, elle vient aussi d'un rapport au réel nourri par l'immédiateté. Et c'est chez Dostoïevski que Guardini trouve la nomenclature de ce rapport de plus ou moins immédiat à l'existence : il y a ceux qui sont silencieux vers leur destinée ; il y a ensuite ceux qui, bien qu'enchaînés à la terre, prennent conscience de leurs chaînes; il y a enfin les hommes de culture. L'existence est destin, conscience et culture ${ }^{8}$. Cette typologie du rapport à Dieu est un essai sur la proximité de Dieu en celui qui souffre. La masse (de ceux qui représentent l'existence-destin) est proche de Dieu sans le savoir : les faits élémentaires de l'existence (la terre, le soleil, les animaux, la souffrance...etc.) ont une saveur religieuse et une valeur symbolique (expriment autre chose sans que cet autre chose ne justifie les expériences par lesquelles on l'atteint). Pour le personnage de Stravoguine, de même que pour Kirilov dans les Possédés, l'athée nietzschéen par excellence, la négation de Dieu revient à projeter sur Dieu la finitude aliénée d'elle-même dans l'angoisse : «Dieu est l'angoisse douloureuse de la mort » dit-il en bon disciple de Feuerbach. Ce geste revient à se prouver qu'on est absolu et qu'on ne peut en même temps disposer de soi de façon achevée que par le suicide, acte humain de désespoir, mais symbole de sa toute-puissance.

\footnotetext{
${ }^{5}$ Guardini, R., La fin des temps modernes, Paris, Le Seuil, 1952, p.111.

${ }^{6}$ Ibid., p.122.

${ }^{7}$ Guardini, R., Royaume de Dieu et liberté de l'homme. Les doutes de l'homme et l'histoire concrète de la foi, Paris, 1960, p.165-166.

${ }^{8}$ Guardini, R., L'univers religieux de Dostö̈evski, Paris, Le Seuil, 1963 (or.1939), p.21.
} 
Guardini identifie, dans son analyse du sacré, une alternative entre une esthétique du oui et une esthétique du non, car la perte de l'expérience religieuse naturelle laisse l'homme devant un choix qui ne repose que sur l'acte de sa volonté propre : il opte pour le consentement ou la resignatio plutôt que pour la toute-puissance. Le sacré, s'il est la perte d'un rapport immanent au divin, est aussi l'apprentissage d'une liberté ontologique. Non pas seulement signe d'une violence originaire, il peut être aussi la nostalgie d'une liberté perdue, la mémoire infime ou la trace qui la fait désirer. Le rapport au sacré est inversé, de violence qu'on est censé combattre, il est aussi le désir de ce qu'on a cru perdu. 